\title{
The CardiAQ transcatheter mitral valve implantation system
}

\author{
Lars Sondergaard ${ }^{1 *}, \mathrm{MD}, \mathrm{MDSc}$; Gian Paolo Ussia², MD, PhD; Nicolas Dumonteil ${ }^{3}, \mathrm{MD}, \mathrm{PhD}$; \\ Arshad Quadri ${ }^{4}, \mathrm{MD}$
}

\begin{abstract}
1. Department of Cardiology, Rigshospitalet, Copenhagen, Denmark; 2. Cardiologia Interventistica Strutturale Policlinico Tor Vergata, Università degli Studi di Roma Tor Vergata, Rome, Italy; 3. Cardiovascular and Metabolic Pole, Rangueil University Hospital, Toulouse, France; 4. CardiAQ Valve Technologies, Irvine, CA, USA
\end{abstract}

This paper also includes accompanying supplementary data published online at: http://www.pcronline.com/eurointervention/W_issue/22

\section{Device description}

Name and manufacturer: The CardiAQ ${ }^{\mathrm{TM}}$ transcatheter mitral valve implantation (TMVI) system; CardiAQ Valve Technologies, Inc., Irvine, CA, USA. The company has been acquired recently by Edwards Lifesciences.

System components: The CardiAQ bioprosthesis, transfemoral and transapical delivery systems.

Approval status: None, investigational device.

Bioprosthesis: Self-expanding nitinol frame, trileaflet bovine tissue, polyester fabric skirt and band, and polyurethane foams (Figure 1).

Frame size: $30 \mathrm{~mm}$ at inflow and $40 \mathrm{~mm}$ at annulus covering native mitral annular dimensions from $36-39.5 \mathrm{~mm}$.

Specific valve design: Symmetric design requires no rotational alignment. Intra- and supra-annular placement of the prosthesis. The frame features two sets of opposing anchors, which secure and align the device with the native mitral annulus. The polyurethane foam-covered left ventricular anchors are designed to engage and preserve the subvalvular apparatus of the mitral valve. The bioprosthesis contains a trileaflet configuration fashioned from bovine pericardial tissue. It also includes polyester fabric skirts on the inflow and outflow aspects of the frame to minimise paraprosthetic leaks. The inflow aspect is reinforced with an additional fabric band to provide further stability during extreme pressure swings. The same bioprosthesis has the ability to be delivered through the transfemoral and transapical approaches using specialised delivery systems.

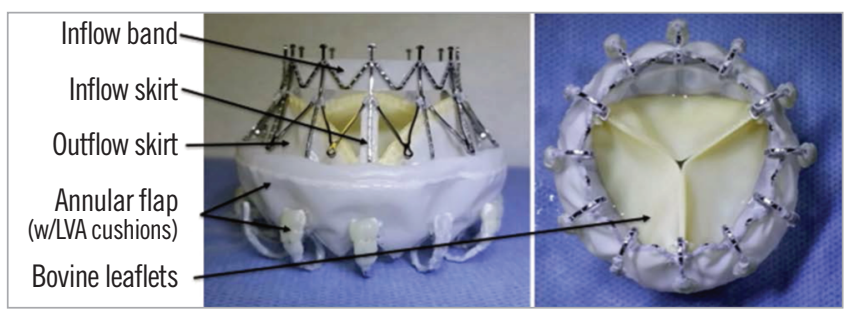

Figure 1. The CardiAQ bioprosthesis. LVA: left ventricular anchors
Delivery systems: Transfemoral delivery system - 33 Fr diameter, $106 \mathrm{~cm}$ working length. Transapical delivery system - 33 Fr diameter, $35 \mathrm{~cm}$ working length. Transapical system contains a lasso control to adjust the diameter of the outflow part of the bioprosthesis.

\section{Procedure details}

After rinsing, the bioprosthesis is loaded to either a transfemoral or a transapical delivery system. After establishing delivery access and wire path, the bioprosthesis implantation may be summarised in four major steps: cross, flip, capture and release (Figure 2).

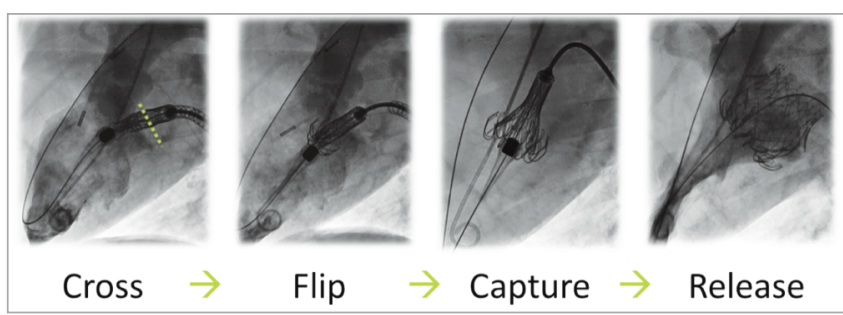

Figure 2. Transfemoral procedure overview.

\section{TRANSFEMORAL ACCESS ROUTE}

Access. To establish a veno-arterial wire path, an intra-atrial septal puncture is performed. An exchange length guidewire is advanced through the left ventricular outflow tract and into the aorta. A snare from the femoral artery is used to grasp the guidewire. The wire path is then checked with a Reliant $46 \mathrm{~mm}$ (Medtronic, Minneapolis, MN, USA) balloon catheter ( $\sim 9 \mathrm{cc}$ in balloon) by advancing it from the left atrium through the left ventricle (Copenhagen manoeuvre). The wire is repositioned in case the balloon reveals engagement with the mitral apparatus. When free of the mitral apparatus, the wire is exchanged for a $500 \mathrm{~cm}$ PTFEcoated nitinol wire, which is externalised from the femoral artery. Cross. Advance the transfemoral delivery system across the septum until the nose cone is in the left atrium. Maintain the guidewire tension on both ends and advance the system into the left ventricle

*Corresponding author: Department of Cardiology, Rigshospitalet, Blegdamsvej 9, 2100 Copenhagen, Denmark. E-mail:drsondergaard@gmail.com 
until the nose cone points towards the aortic valve. Feed the venous guidewire while advancing the system. Once the system begins to cross into the ventricle, feed both ends of the wire simultaneously. After crossing the mitral valve, perform a ventriculogram to reassess the mitral plane. Change the alignment of the system by feeding/retracting the system, and change the height of the system by feeding/retracting the venous wire (arterial wire stays pinned).

Flip. Flip the left ventricular anchors by turning the retraction knob. Retract the nose cone until flush with the bioprosthesis.

Capture. Capture the posterior mitral leaflets by rotating the retraction knob slowly to gain diameter of the bioprosthesis. Continue to rotate the knob fully to gain the diameter, and capture the anterior mitral leaflets and pull the arterial wire if necessary. Confirm the leaflets are captured via transoesophageal echocardiography and fluoroscopy.

Release. Unlock the release lock and rotate the release knob to release the bioprosthesis.

Removal. Centre the system within the bioprosthesis and retract the nose cone until flush with the capsule.

\section{TRANSAPICAL ACCESS ROUTE}

Access. Access the left ventricle through the 5th or 6th intercostal space and use a double purse string on the apex. Place a 0.035 " inch guidewire across the native mitral valve. Perform a Copenhagen balloon manoeuvre to ensure the wire is free of any mitral apparatus.

Cross. Insert the transapical delivery system over the wire and cross the mitral valve until the waist of the left ventricular anchors is even with the native mitral valve (Moving image 1).

Flip. Rotate the capsule knob to retract capsule and flip the left ventricular anchors (Moving image 2).

Capture. Pull the bioprosthesis back into the left ventricle until the left ventricular anchor tips are about $1 \mathrm{~cm}$ below the mitral valve, but above the papillary head, then expand the bioprosthesis using the lasso control. Confirm mitral leaflets are captured by transoesophageal echocardiography and fluoroscopy (Moving image 3, Moving image 4)

Release. Advance the nose cone by rotating the nose cone control knob and confirm the left atrial anchors are above the annulus in the left atrium. Fully advance the nose cone and release the bioprosthesis from the delivery system (Moving image 5).

Removal. Remove the system by centring it within the bioprosthesis and retracting the nose cone until flush with the capsule. Perform final assessment (Moving image 6).

\section{Clinical data}

A FIH case was performed in June 2012 using the first-generation CardiAQ bioprosthesis ${ }^{1}$. From May 2014 to June 2015, there have been 10 attempted implants with the second-generation CardiAQ bioprosthesis. All 10 cases were granted approval through a compassionate access for high or extreme-risk patients. Nine of the cases were through transapical ${ }^{2}$ and one through transfemoral access $^{3}$.

\section{Ongoing and future studies}

In June 2015, CardiAQ initiated its first enrolment in a European safety and performance study for the CardiAQ TMVI system with the transapical delivery system to support a future marketing application for the treatment of moderate to severe mitral valve regurgitation in patients who are considered high or extreme-risk for mortality and morbidity from conventional open heart surgery. The study will attempt to implant the CardiAQ bioprosthesis in up to 40 patients in up to 15 investigational sites.

The US Food and Drug Administration (FDA) has granted approval to perform an Early US Feasibility Study. The trial is expected to initiate enrolment in August 2015 with 20 patients (10 transfemoral and 10 transapical) at up to five investigational sites.

\section{Unique features}

The CardiAQ bioprosthesis design is based on a unique foreshortening frame designed to address the specific challenges of the mitral position, where elasticity and a lack of calcification preclude radial force, where there is minimal space for attachment in the left ventricle and where a risk of obstructing blood flow out of the left ventricle is present. The CardiAQ TMVI system is also truly percutaneous and the access route is transvenous, transseptal and antegrade. It is a single technology/procedure that has the potential to address multiple types of mitral regurgitation and mitigate the challenges of the varying morphology of the disease.

\section{Conflict of interest statement}

L. Sondergaard and G. Ussia have received consultant fees from CardiAQ Valve Technologies. A. Quadri is the founder and coowner of CardiAQ Valve Technologies. N. Dumonteil is a proctor for Edwards LifeSciences, Medtronic and Boston Scientific and a consultant for Biotronik.

\section{References}

1. Søndergaard L, De Backer O, Franzen OW, Holme SJ, Ihlemann N, Vejlstrup NG, Hansen PB, Quadri A. First-in-Human Case of Transfemoral CardiAQ Mitral Valve Implantation. Circ Cardiovasc Interv. 2015;8:e002135.

2. Sondergaard L, Brooks M, Ihlemann N, Jonsson A, Holme S, Tang M, Terp K, Quadri A. Transcatheter mitral valve implantation via transapical approach: an early experience. Eur J Cardiothorac Surg. 2015 Feb 3. [Epub ahead of print].

3. Ussia GP, Quadri A, Cammalleri V, De Vico P, Muscoli S, Marchei M, Ruvolo G, Sondergaard L, Romeo F. Percutaneous transseptal-transfemoral implantation of second generation CardiAQ ${ }^{\mathrm{TM}}$ mitral valve bioprosthesis: first procedure description and 30-day follow-up. EuroIntervention. 2015 Sep 05 [Epub ahead of print].

\section{Online data supplement}

The moving images and legends of this paper can be found online at the following website: http://www.pcronline.com/ eurointervention/W_issue/22. 


\section{Online data supplement}

Moving image 1. Transapical introduction of the delivery system. Moving image 2. Flipping the left ventricular anchors.

Moving image 3. The bioprosthesis is expanded by using the lasso control.

Moving image 4. Mitral leaflet capture confirmed by fluoroscopy. Moving image 5. Release of the bioprosthesis from the delivery system.

Moving image 6. Final ventriculogram to confirm function of bioprosthesis. 